\title{
Palinotaxonomia das espécies de Passiflora L. subg. Decaloba (DC.) Rchb. (Passifloraceae) no Sudeste do Brasil ${ }^{1}$
}

\author{
MICHAELE A. MILWARD-DE-AZEVEDO ${ }^{2,4}$, VANIA GONÇALVES-ESTEVES ${ }^{2}$ e \\ JOSÉ FERNANDO A. BAUMGRATZ ${ }^{3}$
}

(recebido: 4 de junho de 2003; aceito: 22 de julho de 2004)

\begin{abstract}
Palynotaxonomy of Passiflora L. subg. Decaloba (DC.) Rchb. (Passifloraceae) species of the Southeastern Brazil). The palynotaxonomic study of eight species Passiflora L. subg. Decaloba (DC.) Rchb. (Passifloraceae) aimed to contribute to a better characterization, as well as the circumscription and delimitation of this subgenus in Southeastern Brazil. The pollen grains were acetolysed, measured, described, and illustrated using light microscopy. For details observations of surface and aperture, pollen grains not acetolysed were analyzed in scanning electron microscopy (SEM). The data obtained were statistically analyzed according to size of the samples. The species analysed in this study own large or mediun sizes pollen grains, isopolar, prolate spheroidal, spheroidal or subprolate, 12-colporate, 12-colpate for P. suberosa L., or 6-colporate, with pseudopercules, heteroreticulate exine. An identification key with pollinic characters is presented.
\end{abstract}

Key words - Palynology, Passiflora, Passifloraceae, subgenus Decaloba, Southeastern Brazil

RESUMO - (Palinotaxonomia das espécies de Passiflora L. subg. Decaloba (DC.) Rchb. (Passifloraceae) no Sudeste do Brasil). O estudo palinotaxonômico de oito espécies de Passiflora L. subg. Decaloba (DC.) Rchb. (Passifloraceae) teve como objetivo contribuir para a caracterização, circunscrição e delimitação desse subgênero na região Sudeste do Brasil. Os grãos de pólen foram acetolisados, medidos, descritos e ilustrados sob microscopia de luz. Para observar detalhes da superfície e da abertura, grãos de pólen não acetolisados foram analisados em microscópio eletrônico de varredura (MEV) e, em seguida, eletromicrografados. Os dados obtidos foram estatisticamente analisados, de acordo com o tamanho da amostra. As espécies analisadas neste estudo possuem grãos de pólen grandes ou médios, isopolares, prolato-esferoidais, esferoidais ou subprolatos, 12-colporados, 12-colpados para P. suberosa L. ou 6-colporados, com pseudopérculos, exina heteroreticulada. Foi confeccionada uma chave para a identificação das espécies estudadas com base em dados polínicos.

Palavras-chave - Palinologia, Passiflora, Passifloraceae, subgênero Decaloba, Sudeste brasileiro

\section{Introdução}

Passiflora L. subg. Decaloba (DC.) Rchb. (= subg. Plectostemma Mast.) possui aproximadamente 130 espécies distribuídas em oito seções e é caracterizada por possuir inflorescências paucifloras, flores freqüentemente pequenas, brancas ou amareladas, hipanto pateliforme ou campanulado dividido em 10 lobos, corona com uma ou duas séries de filamentos, opérculo plicado e filetes unidos na base e

1. Parte da dissertação de M.A. Milward-de-Azevedo, Programa de Pós-Graduação em Ciências Biológicas (Botânica), Museu Nacional/UFRJ

2. Universidade Federal do Rio de Janeiro, Museu Nacional, Departamento de Botânica, Laboratório de Palinologia, Quinta da Boa Vista, São Cristóvão, 20940-040 Rio de Janeiro, RJ, Brasil.

3. Instituto de Pesquisas Jardim Botânico do Rio de Janeiro, Rua Pacheco Leão 915, Jardim Botânico, 22460-030 Rio de Janeiro, RJ, Brasil.

4. Autor para correspondência: michaelemilward@aol.com encurvados para o interior da flor, em direção ao ápice do ovário.

As Passifloraceae encontram-se diversificadas na flora brasileira e estruturadas em um sistema de classificação complexo, baseado em Killip (1938), pouco se conhecendo sobre a importância da morfologia polínica na caracterização das espécies do gênero Passiflora, principalmente, sobre as características que possam ter valor taxonômico e auxiliar a sistemática do gênero.

Presting (1965) descreveu os grãos de pólen de 153 espécies pertencentes a 13 gêneros, apresentando um estudo aprofundado do sistema apertural e propondo uma filogenia para a família, baseado em características polínicas. Spirlet (1965) associou a sistemática usual do gênero Passiflora com a morfologia dos grãos de pólen, mostrando que essa classificação segue as propostas evolutivas que têm sido apresentadas sobre as Passifloraceae. Huynh (1972) estudou o arranjo dos micrósporos no estágio de tétrade pós-meiótico mostrando que o gênero Passiflora pode apresentar, num mesmo grão de pólen, mesocolpos de diferentes dimensões. 
Huang (1968) analisou grãos de pólen de várias famílias da flora de Formosa, Taiwan e, dentre elas, três espécies de Passiflora.

O presente trabalho objetiva a caracterização morfopalinológica e a identificação de caracteres de valor diagnóstico que contribuam para a taxonomia das oito espécies deste subgênero ocorrentes na região Sudeste do Brasil, pertencentes aos seguintes táxons: seção Cieca - Passiflora suberosa L., P. truncata Regel; seção Decaloba série Organensis P. organensis Gardn.; seção Decaloba série Punctatae - P. pohlii Mast.; seção Decaloba série Miserae P. misera Kunth, P. tricuspis Mast.; seção Pseudodysosmia - P. morifolia Mast. e seção Xerogona - P. capsularis L.

\section{Material e métodos}

O material polínico foi obtido de botões florais de exemplares depositados nos Herbários: BHCB, GUA, HB, HRCB, IAC, PAMG, R, RB, SP, SPF e UEC, utilizando-se material coletado não só na região Sudeste, como em outras regiões do Brasil, quando se tratava do único exemplar com botões florais disponíveis. Essas siglas seguem o Index Herbariorum (Holmgren et al. 1990).

Para cada espécie foi estudado um mínimo de três espécimes, sendo um deles considerado como padrão, indicado por asterisco junto à sigla do herbário depositário, e os outros como comparação. Segue a relação do material estudado: Passiflora capsularis - BRASIL: Espírito SANTO: Goytacazes, 8-XI-1943, J.G. Kuhlmann 6472 (RB*). MinAS GERAIS: s.loc., 24-V-1984, P.M. Andrade \& M.A. Lopes 247 (RB). São PAUlo: Bom Sucesso de Itararé, 11-XII-1997, J.M. Torezan et al. 538 (UEC); Jundiaí, 11-XI-1996, L.C. Bernacci 2178 (IAC); ibidem, 26-II-1999, S.L. Jung-Mendaçolli et al. 974 (IAC). P. misera - BRASIL: EspírIto SANTo: Vitória, 19-V-1983, N.C. Maciel \& D. Araújo 47 (GUA*). Minas GeraIs: Pouso Alegre, 28-IV-1927, F.C. Hoehne s.n. (SP19242, SPF146570). Paraná: São Mateus do Sul, 16-XII-1969, G. Hatschbach 23275 (RB). São Paulo: Campinas, 6-XI-1938, C. Franco \& P. Mendes s.n. (IAC2851); São Paulo, 9-II-1942, L. Roth 835 (SP); Presidente Epitácio, 23-XI-1992, I. Cordeiro et al. 1133 (SP, SPF). P. morifolia - BRASIL: MINAS GERAIS: Viçosa, 11-III-1985, G.L.F.S. Lopes s.n.(PAMG38911, VIC9304). São PAulo: Águas de Prata, 21-III-1994, A.B. Martins et al. 31478 (SPF, UEC*); Rio Claro, 28-III-1978, Pagano \& Saitori 9 (HRCB, UEC); São Paulo, 21-III-1945, W. Hoehne s.n. (IAC32621, SPF11470). P. organensis - BRASIL: EsPíRITO SANTO: Santa Teresa, 13-II-1999, R. Mello-Silva et al. 1575 (SPF). Rio de JANEIRo: Angra dos Reis, 11-III-1986, D. Araújo et al. 7279 (GUA*); ibidem, 1-III-1965, Lanna \& Castellanos 835 (GUA); Santa Maria Madalena, 21-III-1989, R. Marquete et al. 211 (RB). São PAULO: São Paulo, 25-II-1972, O. Handro
2200 (HB). P. pohlii - BRASIL: EsPíRITO SANTO: Linhares, 20-III-1960, J. Delistoianov s.n. (IAC18582). GoIÁs: Niquelândia, 17-IX-1996, M.A. Silva et al. 3074 (RB). MinAS GERAIs: Belo Horizonte, 19-IX-1945, O. Williams \& V. Assis 7577 (R*); Curvelo, 11-X-1998, R.M. Harley et al. 24805 (SPF); Prudente de Morais, 2-IV-1998, J.F. Macedo 2561 (PAMG). P. suberosa - BRASIL: MinAs GERAIs: Lavras, 9-XII-1980, H.F. Leitão et al. 11697 (UEC); Paraopeba, 22-VI-1955, E.P. Heringer s.n. (RB90516). SÃo PAULO: Jundiaí, 25-X-1994, L.C. Bernacci 2221 (IAC); São Paulo, 11-IV-1935, M. Kuhlmann s.n. (SPF146576); ibidem, 18-IV-1967, N. Mazzaro s.n. (IAC19481*). P. tricuspis - BRASIL: MATO Grosso do Sul: Antônio João, 27-I-2001, M. Groppo Jr. et al. 578 (SPF). Minas Gerais: Pitangui, 9-X-2001, F.C. Campos Neto s.n. (BHCB64897). PARANÁ: Faxinal, 16-XI-1969, G. Hatschbach 22883 (UEC*). SAnta CATARINA: Nova Teotônia, 28-III-1944, F. Plaumann 436 (RB). P. truncata - BRASIL: RIO DE JANEIRO: Mendes, 6-III-1980, M.B. Cosori et al. 166 (GUA); Rio de Janeiro, 22-I-1932, Brade s.n. (R90277). SÃo PAUlo: São Paulo, 28-XI-1980, F. Barros \& S.L. Jung-Mendaçolli 548, (IAC, SP, UEC*); ibidem, 23-XI-1972, O. Handro 2216 (RB, SPF).

Os grãos de pólen foram acetolisados (Erdtman 1960), medidos até sete dias após sua preparação (SalgadoLabouriau 1973), os dados quantitativos, submetidos a tratamento estatístico sendo, posteriormente, fotomicrografados. Nas amostras do material padrão foram medidos, aleatoriamente, o diâmetro polar (DP) e o diâmetro equatorial (DE) de 25 grãos de pólen em vista equatorial, calculando-se a média aritmética $(\overline{\mathrm{x}})$, o desvio padrão da amostra (s), o desvio padrão da média $\left(\mathrm{s}_{\mathrm{x}}\right)$, o coeficiente de variabilidade (CV\%) e o intervalo de confiança a $95 \%$ (Vieira 1981). Para análise em microscópio eletrônico de varredura (MEV), as anteras foram maceradas e os grãos de pólen não acetolisados foram pulverizados sobre suportes específicos recobertos por fita de carbono. O conjunto foi metalizado com uma camada de ouro puro por ca. 3 minutos e eletromicrografados em microscópio JSM-5310, pertencente ao laboratório de Biofísica da UFRJ.

Foram realizadas para cada espécie, na amostra padrão, 10 medidas da abertura (comprimento e largura) quando em vista equatorial e, quando em vista polar, do diâmetro do lúmen, do diâmetro equatorial (DEVP) e do lado do apocolpo (LA), bem como das camadas da exina (sexina e nexina). A medida da exina foi feita sempre com o grão de pólen em vista polar, na região mediana do mesocolpo. Nesses casos, foi apenas calculada a média aritmética.

A terminologia adotada seguiu Barth \& Melhem (1988) e Punt et al. (1999). Para a descrição geral das aberturas foi adotada a definição de Presting (1965), onde o termo pseudopérculo é usado para descrever a estrutura ornamentada resultante do concrescimento aos pares dos mesocolpos e colpos. O pseudopérculo não tem apenas a aparência de um opérculo mas, segundo o autor, também, às vezes, funciona como opérculo. Essa terminologia não foi, 
ainda, definida nos glossários palinológicos utilizados no presente estudo.

Para as amostras do material de comparação foram realizadas 10 medidas do diâmetro polar (DP) e do diâmetro equatorial (DE) em vista equatorial, calculando-se a média aritmética.

\section{Resultados}

As espécies de Passiflora subg. Decaloba analisadas neste estudo (figuras 1-59) apresentaram grãos de pólen médios ou grandes (na maioria das espécies), isopolares, prolato-esferoidais (na maioria das espécies), esferoidais ou subprolatos (tabelas 1,2), âmbito circular, área polar muito pequena (na maioria das espécies) ou pequena (tabela 3), 12-colporados na maioria das espécies (figuras 1, 7, 15, 21, 28, 43), 12-colpados em P. suberosa (figura 36) e 6-colporados em $P$. truncata (figura 52), colpos muito longos a longos, com pseudopérculos (3 ou 6), endoaberturas lolongadas, exina heterorreticulada.

O comprimento dos colpos variou de $38,0 \mu \mathrm{m}$ (em P. truncata) a 52,7 $\mu \mathrm{m}$ (em P. pohlii); quanto à largura, os colpos foram considerados estreitos na maioria das espécies, sendo mais largos em $P$. suberosa. Nos grãos de pólen colporados, o comprimento da endoabertura, na maioria das espécies, foi cerca de $10 \mu \mathrm{m}$; apenas em $P$. morifolia $\mathrm{o}$ valor foi de $16,0 \mu \mathrm{m}$; quanto à largura, $\mathrm{o}$ menor valor $(4,0 \mu \mathrm{m})$ foi encontrado em $P$. misera e o maior valor $(8,7 \mu \mathrm{m})$, em $P$. truncata (tabela 4$)$.

O retículo apresentou diferenças nas dimensões de seus muros e lumens entre os grãos de pólen estudados (tabela 5). Muros descritos como sinuosos, altos (ca. 0,7 $\mu \mathrm{m}$ ) e estreitos formando lumens grandes com muitos báculos no seu interior foram encontrados em P. capsularis (figura 6) e P. morifolia (figura 20). Na maioria das espécies, os muros foram retos, baixos (ca. 0,2 $\mu \mathrm{m}$ ) e largos, formando lumens grandes ou pequenos, com poucos báculos (P. misera, $P$. pohlii e $P$. tricuspis) ou sem báculos ( $P$. organensis, $P$. suberosa e $P$. truncata) no interior dos lumens. Entre as espécies estudadas, $P$. truncata apresentou grãos de pólen com retículos formados por muros largos e os menores lumens (figura 59). Em todas as espécies, a sexina foi simplescolumelada e mais espessa que a nexina.

Os materiais usados como comparação dos resultados (tabela 6) mantiveram-se, em sua maioria, fora dos limites do intervalo de confiança e/ou da faixa de variação, quando comparados com o respectivo material padrão, demonstrando a grande variabilidade existente nas dimensões dos grãos de pólen.

Chave polínica para identificação das espécies estudadas de Passiflora subg. Decaloba

(A = seção Cieca $; \mathrm{B}=$ seção Xerogona $; \mathrm{C}=$ seção Pseudodysosmia $; \mathrm{D}_{1}=$ seção Decaloba série Organensis; $\mathrm{D}_{2}=$ seção Decaloba série Punctatae $; \mathrm{D}_{3}=$ seção Decaloba série Miserae)

1. Grãos de pólen colpados

P. suberosa $(\mathrm{A})$

1. Grãos de pólen colporados

2. Grãos de pólen 6-colporados P. truncata (A)

2. Grãos de pólen 12-colporados

3. Retículos com muros sinuosos, altos e estreitos

4. Média do diâmetro dos lumens do retículo $<10,0 \mu \mathrm{m}$ P. capsularis $(\mathrm{B})$

4. Média do diâmetro dos lumens do retículo $\geq 10,0 \mu \mathrm{m}$ P. morifolia (C)

3. Retículos com muros retos, baixos e largos

5. Lumens do retículo sem báculos no interior... P. organensis $\left(\mathrm{D}_{1}\right)$

5. Lumens do retículo com poucos báculos no interior

6. Média do comprimento do colpo $>50,0 \mu \mathrm{m}$. P. pohlii $\left(\mathrm{D}_{2}\right)$

6. Média do comprimento do colpo $<40,0 \mu \mathrm{m}$

7. Média do diâmetro dos lumens do retículo $>3,5 \mu \mathrm{m}$ P. tricuspis $\left(\mathrm{D}_{3}\right)$

7. Média do diâmetro dos lumens do retículo $<3,0 \mu \mathrm{m}$ P. misera $\left(\mathrm{D}_{3}\right)$ 

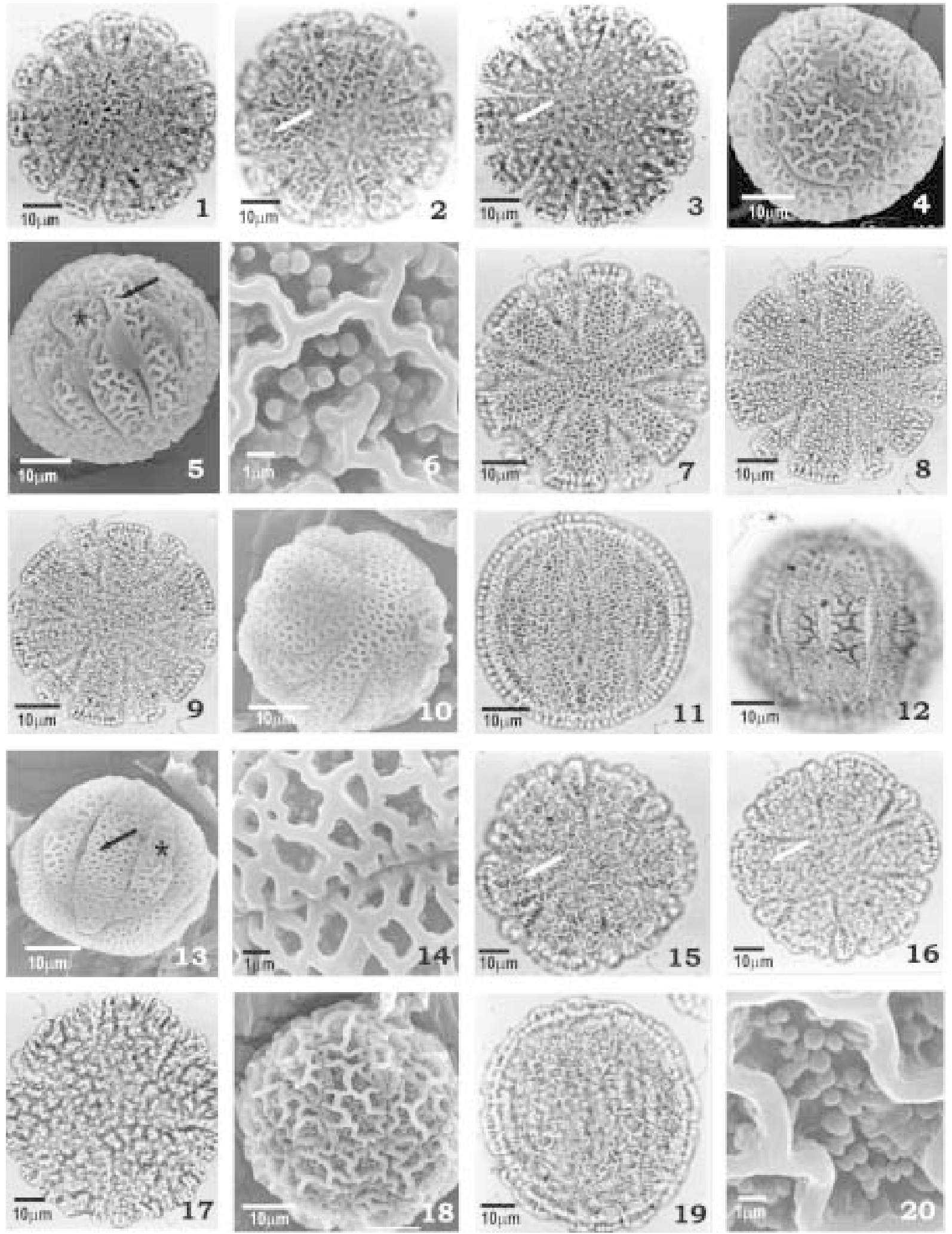

Figuras 1-20. Fotomicrografias e eletromicrografias dos grãos de pólen de espécies de Passiflora: 1-6. P. capsularis, vista polar: 1. Corte óptico. 2-3. Análise de L.O. (setas). 4. Apocolpo. Vista equatorial: 5. Aberturas, mesocolpo (seta) e pseudopérculo (asterisco). 6. Detalhe da superfície. 7-14. P. misera, vista polar: 7-8. Análise de L.O. 9. Corte óptico. 10. Apocolpo e superfície. Vista equatorial: 11. Corte óptico. 12-13. Abertura, mesocolpo (seta) e pseudopérculo (asterisco). 14. Detalhe da superfície. 15-20. P. morifolia, vista polar: 15-16. Análise de L.O. (seta). 17. Corte óptico. 18. Apocolpo e superfície. Vista equatorial: 19. Corte óptico. 20. Detalhe da superfície. 
Tabela 1. Caracterização morfológica dos grãos de pólen $(\mathrm{n}=25)$ de espécies de Passiflora subg. Decaloba.

Table 1. Morphologic caracterization of pollen grains $(\mathrm{n}=25)$ of species of Passiflora subg. Decaloba.

\begin{tabular}{lllllc}
\hline Espécies & Tamanho & \multicolumn{1}{c}{ Forma } & DP/DE & Abertura & Figuras \\
\hline P. capsularis & grande & prolato-esferoidal & 1,05 & 12-colporado & $1-6$ \\
P. misera & médio & prolato-esferoidal & 1,02 & 12-colporado & $7-14$ \\
P. morifolia & grande & prolato-esferoidal & 1,12 & 12-colporado & $15-20$ \\
P. rganensis & grande & prolato-esferoidal & 1,03 & 12-colporado & $21-27$ \\
P. pohlii & grande & prolato-esferoidal & 1,10 & 12-colporado & $28-35$ \\
P. suberosa & grande & subprolato & 1,21 & 12-colpado & $36-42$ \\
P. tricuspis & grande & prolato-esferoidal & 1,05 & 12-colporado & $43-51$ \\
P. truncata & grande & prolato-esferoidal & 1,08 & 6-colporado & $52-59$ \\
\hline
\end{tabular}

\section{Discussão}

Presting (1965) descreveu os grãos de pólen de 153 espécies de Passifloraceae, entre elas P. capsularis, P. misera, P. morifolia, P. organensis, $P$. pohlii e $P$. suberosa, fornecendo um estudo profundo do sistema apertural e propondo uma filogenia para a família baseada em caracteres polínicos. Para as espécies aqui estudadas e também analisadas por esse autor, os grãos de pólen foram caracterizados como 6-colporados (P. misera, $P$. morifolia, $P$. organensis e $P$. suberosa) ou 12-colporados ( $P$. capsularis e P. pohlii), com os colpos livres, tendendo ao concrescimento aos pares ou de quatro em quatro em P. capsularis. Os resultados obtidos no presente estudo foram semelhantes aos encontrados por Presting (1965) no que se refere ao formato do retículo, às características do lúmen e da exina. O número e tipo de abertura de $P$. capsularis e $P$. pohlii também foram semelhantes porém, $P$. suberosa foi aqui descrita como possuindo grãos de pólen 12-colpados, enquanto $P$. misera, P. morifolia e P. organensis, como 12-colporados.

Huang (1968) apresentou breves descrições polínicas de três espécies de Passiflora. Os grãos de pólen de Passiflora suberosa foram considerados, pelo autor, como sendo 6-colpados, com exina intectada, clavada ou tectada, com teto possuindo processos verrucosos. No presente trabalho, o tipo de abertura foi a única característica encontrada para $P$. suberosa que está de acordo com os resultados apresentados por Huang (1968).

Huynh (1972) estudou o arranjo dos grãos de pólen de Passiflora ainda na fase de tétrade, procurando relacionar a organização dos colpos e mesocolpos em grãos de pólen livres com aqueles da tétrade pósmeiótica. Nesse trabalho, o autor analisou cinco espécies, duas das quais ( $P$. capsularis e $P$. suberosa) também foram aqui estudadas. Esse autor considerou que $P$. capsularis possuía 12 cólporos estreitos, com endoaberturas muito estreitas, enquanto $P$. suberosa possuía grãos de pólen com seis cólporos. Segundo o autor, o mesmo grão de pólen podia apresentar diferentes tamanhos de mesocolpo e esse fato estaria relacionado com a fase de tétrade, quando os micrósporos de cada par de micrósporos-irmãos estariam posicionados diametralmente opostos a um mesocolpo mais largo. Os resultados encontrados no presente estudo para P. capsularis foram semelhantes aos de Huynh (1972), no que se refere ao número e tipo de abertura embora, para $P$. suberosa, os grãos de pólen tenham sido agora caracterizados como 12-colpados. A correlação com a fase de tétrade não pôde ser realizada no presente estudo.

Roubik \& Moreno (1991) realizaram um estudo polínico de espécies de várias famílias ocorrentes na

Figures 1-20. Photomicrographs by light microscopy and scanning electron microscopy of pollen grains of Passiflora species: 1-6. P. capsularis, Polar view: 1. Cross section. 2-3. L.O analysis (arrows). 4. Apocolpium. Equatorial view: 5. Aperture, mesocolpium (arrow) and pseudoperculum (asterisk). 6. Detail of surface. 7-14. P. misera, Polar view: 7-8. L.O. analysis. 9. Cross section. 10. Apocolpium. Equatorial view: 11. Cross section. 12-13. Aperture, mesoculpium (arrow) and pseudoperculum (asterisk). 14. Detail of surface. 15-20. P. morifolia, Polar view: 15-16. L.O. analysis (arrows). 17. Cross section. 18. Apocolpium. Equatorial view: 19. Cross section. 20. Detail of surface. 

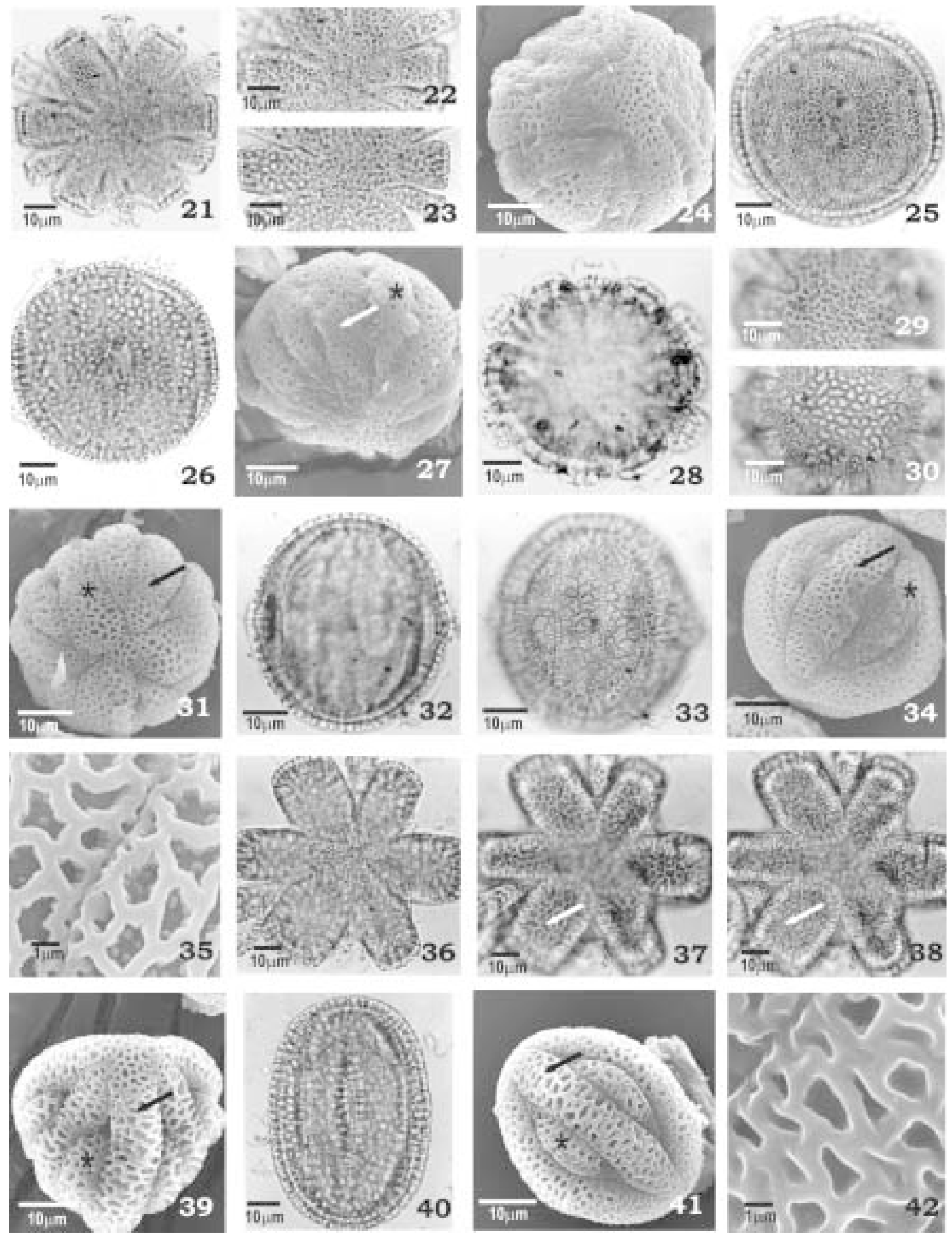

Figuras 21-42. Fotomicrografias e eletromicrografias dos grãos de pólen de espécies de Passiflora: 21-27. P. organensis, vista polar: 21. Corte óptico, mesocolpo (seta) e pseudopérculo (asterisco). 22-23. Análise de L.O. 24. Apocolpo e superfície. Vista equatorial: 25. Corte óptico. 26-27. Abertura, mesocolpo (seta) e pseudopérculo (asterisco). 28-35. P. pohlii, vista polar: 28. Corte óptico. 29-30. Análise de L.O. 31. Apocolpo, mesocolpo (seta) e pseudopérculo (asterisco). Vista equatorial: 32. Corte óptico. 33-34. Abertura, mesocolpo (seta) e pseudopérculo (asterisco). 35. Detalhe da superfície. 36-42. P. suberosa, vista polar: 36. Corte óptico. 37-38. Análise de L.O. (setas). 39. Apocolpo, mesocolpo (seta) e pseudopérculo (asterisco). Vista equatorial: 40. Corte óptico e abertura. 41. Abertura, mesocolpo (seta) e pseudopérculo (asterisco). 42. Detalhe da superfície. 
Tabela 2. Medidas (em $\mu \mathrm{m})$ dos grãos de pólen em vista equatorial $(\mathrm{n}=25)$ de espécies de Passiflora subg. Decaloba $. \overline{\mathrm{x}}=$ média aritmética; $\mathrm{s}_{\mathrm{x}}=$ desvio padrão da média; $\mathrm{s}=$ desvio padrão da amostra; I.C. = intervalo de confiança; $\mathrm{CV}=$ coeficiente de variabilidade.

Table 2. Measures $(\mu \mathrm{m})$ of pollen grains in equatorial view $(\mathrm{n}=25)$ of species of Passiflora subg. Decaloba. $\overline{\mathrm{x}}=$ arithmetic mean; $\overline{\mathrm{x}}=$ standard of medium; $\mathrm{s}=$ standard of sample; I.C. = interval of confidence; $\mathrm{CV}=$ level of variability.

\begin{tabular}{|c|c|c|c|c|c|c|c|c|c|c|}
\hline \multirow[t]{2}{*}{ Espécies } & \multicolumn{5}{|c|}{ Diâmetro polar } & \multicolumn{5}{|c|}{ Diâmetro equatorial } \\
\hline & Faixa de variação & $\overline{\mathrm{X}} \pm \mathrm{s}_{\mathrm{x}}$ & $\mathrm{s}$ & I.C. $95 \%$ & $\mathrm{CV} \%$ & Faixa de variação & $\bar{x} \pm s_{x}$ & $\mathrm{~s}$ & I.C. $95 \%$ & $\mathrm{CV} \%$ \\
\hline P. capsularis & $48,0-60,0$ & $54,3 \pm 0,6$ & 3,0 & $53,1-55,5$ & 5,5 & $46,0-56,0$ & $51,7 \pm 0,6$ & 3,1 & $50,5-52,9$ & 6,0 \\
\hline P. misera & $44,0-50,0$ & $46,6 \pm 0,4$ & 2,0 & $45,8-47,4$ & 4,3 & $41,0-50,0$ & $45,9 \pm 0,5$ & 2,5 & $44,9-46,9$ & 5,4 \\
\hline P. morifolia & $57,5-67,5$ & $62,3 \pm 0,8$ & 3,9 & $60,5-64,0$ & 6,3 & $50,0-62,5$ & $55,7 \pm 1,1$ & 5,1 & $45,3-66,2$ & 9,1 \\
\hline P. organensis & $54,0-61,0$ & $56,9 \pm 0,4$ & 2,0 & $56,1-57,7$ & 3,5 & $52,0-60,0$ & $55,3 \pm 0,5$ & 2,5 & $54,3-56,3$ & 4,5 \\
\hline P. pohlii & $56,0-59,5$ & $57,3 \pm 0,2$ & 1,0 & $56,9-57,7$ & 1,7 & $49,0-55,0$ & $52,4 \pm 0,3$ & 1,7 & $51,8-53,0$ & 3,2 \\
\hline P. suberosa & $57,5-77,5$ & $66,3 \pm 1,2$ & 5,8 & $63,9-68,7$ & 8,8 & $47,5-60,0$ & $54,8 \pm 0,8$ & 3,9 & $53,2-56,4$ & 7,2 \\
\hline P. tricuspis & $52,5-57,5$ & $54,7 \pm 0,3$ & 1,6 & $51,4-58,0$ & 2,9 & $45,0-55,0$ & $51,7 \pm 0,5$ & 2,7 & $46,1-57,3$ & 5,2 \\
\hline P. truncata & $47,5-52,5$ & $50,4 \pm 0,4$ & 1,9 & $49,6-51,2$ & 3,9 & $45,0-50,0$ & $46,7 \pm 0,4$ & 2,0 & $45,9-47,5$ & 4,2 \\
\hline
\end{tabular}

Tabela 3. Médias (em $\mu \mathrm{m})$ do diâmetro equatorial (DEVP), lado do apocolpo (LA) e índice de área polar (IAP), em vista polar $(\mathrm{n}=10)$ de espécies de Passiflora subg. Decaloba.

Table 3. Means $(\mu \mathrm{m})$ of equatorial diameter (DEVP), apocolpium (LA) and polar area (IAP) in polar view $(\mathrm{n}=10)$ of Passiflora subg. Decaloba species.

\begin{tabular}{llrl}
\hline Espécies & DEVP & LA & IAP \\
\hline P. capsularis & 58,0 & 10,0 & 0,17 \\
P. misera & 47,2 & 9,8 & 0,21 \\
P. morifolia & 67,2 & 18,0 & 0,27 \\
$P$. organensis & 65,8 & 11,6 & 0,18 \\
$P$. pohlii & 60,2 & 12,5 & 0,21 \\
$P$. suberosa & 76,2 & 20,9 & 0,27 \\
$P$. tricuspis & 53,9 & 9,0 & 0,17 \\
$P$. truncata & 52,5 & 10,5 & 0,20 \\
\hline
\end{tabular}

Ilha de Barro Colorado, Panamá. Dentre as famílias estudadas, Passifloraceae foi objeto de interesse, com 11 espécies do gênero Passiflora. Para esses autores, o grão de pólen típico da família apresentou vista polar trirradial, com 3-9 pré-opérculos que, segundo os autores, são áreas livres nos intercolpos. Esses pré-opérculos são formados pela união de pares de colpos adjacentes, parecendo um anel longitudinal contínuo. No presente estudo, o termo pré-opérculo sensu Roubik \& Moreno (1991) foi considerado como pseudopérculo (sensu Presting 1965). Quanto às demais características

Tabela 4. Média (em $\mu \mathrm{m}$ ) das medidas das aberturas do pólen (n = 10) de espécies de Passiflora subg. Decaloba.

Table 4. Mean $(\mu \mathrm{m})$ of pollen grains apertures $(n=10)$ of Passiflora subg. Decaloba species.

\begin{tabular}{lccccc}
\hline \multirow{2}{*}{ Espécies } & \multicolumn{2}{c}{ Colpo } & & \multicolumn{2}{c}{ Endoabertura } \\
\cline { 2 - 3 } \cline { 6 - 6 } & compr. & larg. & & compr. & larg. \\
\hline P. capsularis & 44,8 & 1,2 & & 10,7 & 6,5 \\
P. misera & 39,5 & 1,3 & & 10,1 & 4,0 \\
$P$. morifolia & 43,7 & 2,5 & & 16,0 & 7,5 \\
$P$. organensis & 42,3 & 1,0 & & 9,6 & 7,2 \\
$P$. pohlii & 52,7 & 1,1 & & 12,3 & 7,7 \\
$P$. suberosa & 50,0 & 3,7 & & - & - \\
$P$. tricuspis & 39,2 & 2,5 & & 10,7 & 7,7 \\
$P$. truncata & 38,0 & 2,5 & & 12,2 & 8,7 \\
\hline
\end{tabular}

Figures. 21-42. Photomicrographs by light microscopy and scanning electron microscopy of pollen grains of Passiflora species: 21-27. P. organensis, Polar view: 21. Cross section, mesocolpium (arrow) and pseudoperculum (asterisk). 22-23. L.O. analysis. 24. Apocolpium. Equatorial view: 25. Cross section. 26-27. Aperture, mesoculpium (arrow) and pseudoperculum (asterisk). 28-35. P. pohlii, Polar view: 28. Cross section. 29-30. L.O. analysis. 31. Apocolpium, mesocolpium (arrow) and pseudoperculum (asterisk). Equatorial view: 32. Cross section. 33-34. Aperture, mesocolpium (arrow) and pseudoperculum (asterisk). 35. Detail of surface. 36-42. P. suberosa, Polar view: 36. Cross section. 37-38. L.O. analysis. 39. Apocolpium, mesocolpium (arrow) and pseudoperculum (asterisk). Equatorial view: 40. Aperture and cross section. 41. Aperture, mesocolpium (arrow) and pseudoperculum (asterisk). 42. Detail of surface. 

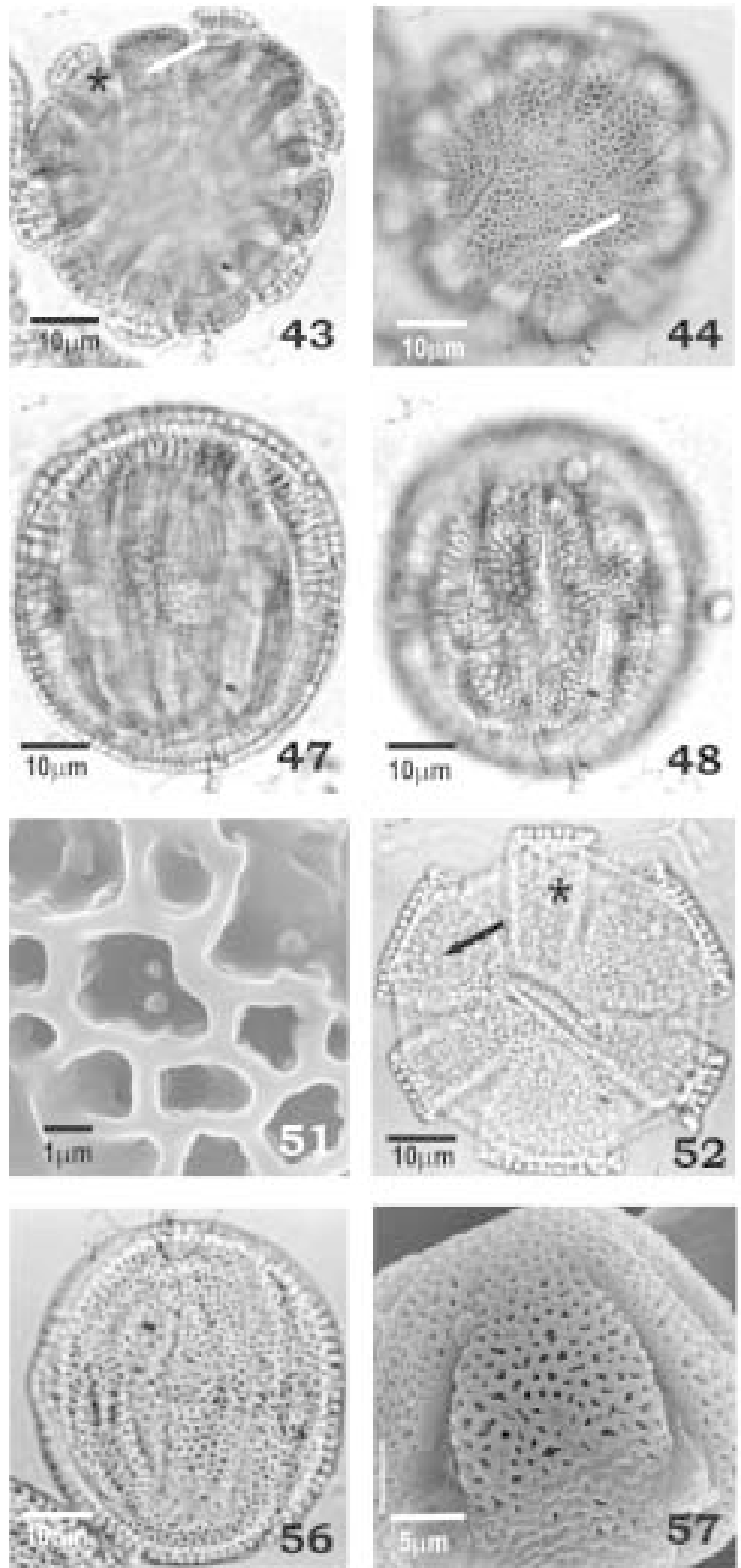
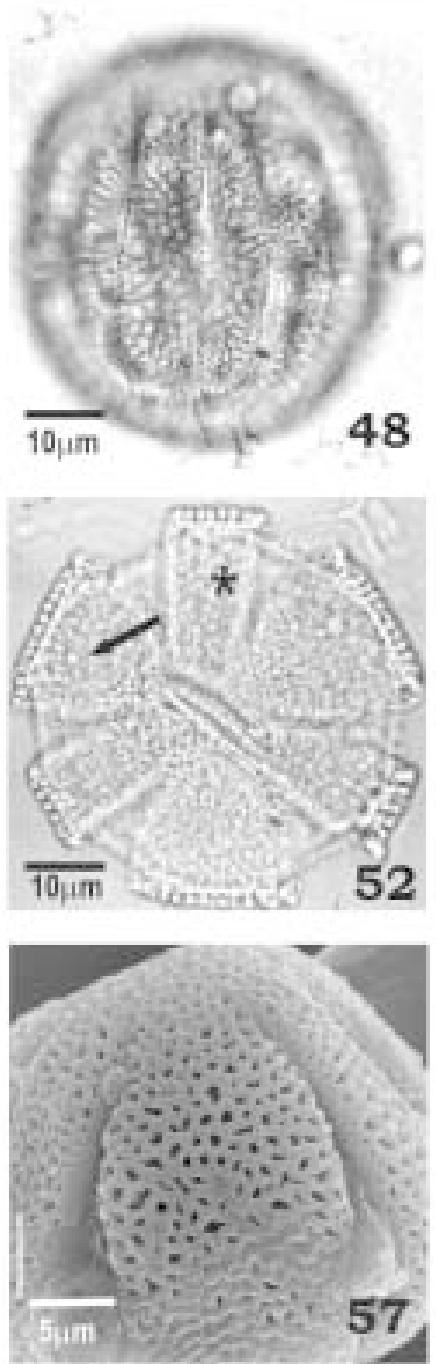
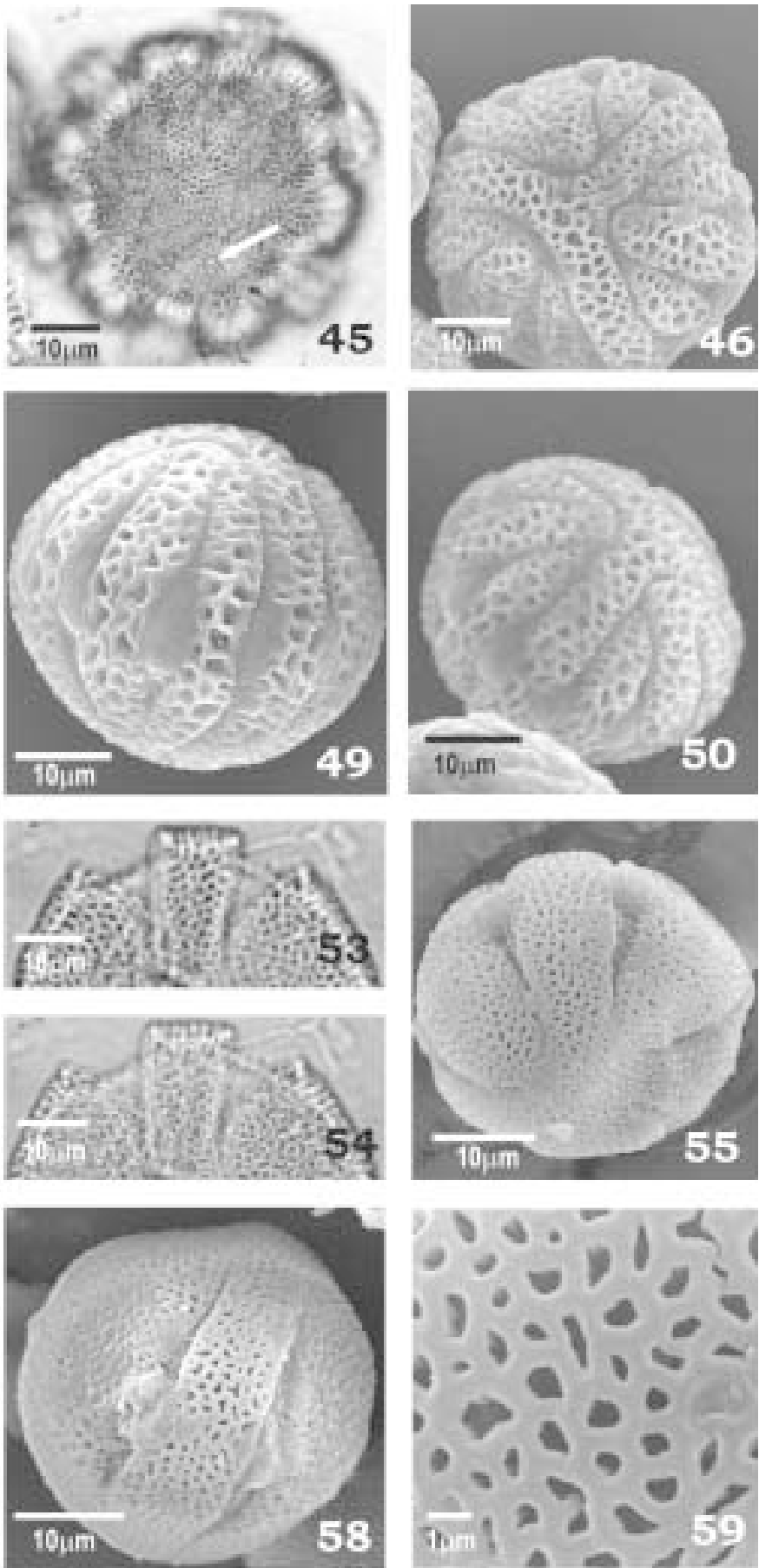

Figuras 43-59. Fotomicrografias e eletromicrografias dos grãos de pólen de espécies de Passiflora: 43-51. P. tricuspis, vista polar: 43. Corte óptico, mesocolpo (seta) e pseudopérculo (asterisco). 44-45. Análise de L.O. (setas). 46. Apocolpo. Vista equatorial: 47. Corte óptico. 48-49. Abertura. 50. Detalhe do mesocolpo. 51. Detalhe da superfície. 52-59. P. truncata, vista polar: 52. Corte óptico, mesocolpo (seta) e pseudopérculo (asterisco). 53-54. Análise de L.O. 55. Apocolpo. Vista equatorial: 56. Corte óptico. 57. Detalhe do mesocolpo. 58. Abertura. 59. Detalhe da superfície.

Figures 43-59. Photomicrographs by light microscopy and scanning electron microscopy of pollen grains of Passiflora species: 43-51. P. tricuspis, Polar view: 43. Cross section, mesocolpium (arrow) and pseudoperculum (asterisk). 44-45. L.O. analysis (arrows). 46. Apocolpium. Equatorial view: 47. Cross section. 48-49. Aperture. 50. Detail of mesocolpium. 51. Detail of surface. 52-59. P. truncata, Polar view: 52. Cross section, mesocolpium (arrow) and pseudoperculum (asterisk). 53-54. L.O. analysis. 55. Apocolpium. Equatorial view: 56. Cross section. 57. Detail of mesocolpium. 58. Aperture. 59. Detail of surface. 
descritas por esses autores para as espécies de Passiflora, na sua maioria também foram aqui observadas, apesar de se tratar de outras espécies.

MacDougal (1994) fez a revisão do subgênero Decaloba seção Pseudodysosmia (Harms) Killip, estudando 18 espécies e dentre elas, $P$. morifolia. Esse autor descreveu os grãos de pólen como oblatos a esferoidais, 6-colporados a 6-sincolporados, fortemente reticulados, com "columelas comuns expostas" (= báculos no interior dos lumens) e com, aproximadamente, 40-65 $\mu \mathrm{m}$ de diâmetro. Os resultados obtidos no presente trabalho foram semelhantes aos encontrados por MacDougal (1994) no que se refere ao tamanho dos grãos de pólen, às características do retículo, lúmen e da exina, porém difere na quantidade de cólporos e na forma encontrada para $P$. morifolia que foi descrita, no presente estudo, como prolato-esferoidal e subprolata no material de comparação.

García et al. (2002), analisando a ontogenia e a palinologia de seis espécies de Passiflora pertencentes a três subgêneros - Passiflora, Dysosmia e Decaloba, estudaram os grãos de pólen de $P$. misera e $P$. suberosa. Segundo os autores, as medidas polínicas $(\mathrm{n}=10)$ foram realizadas em microscópio de luz, com grãos de pólen não acetolisados, montados apenas em água glicerinada, pois o material submetido à acetólise variou consideravelmente na forma. Os resultados encontrados por esses autores diferem daqueles aqui observados, uma vez que os grãos de pólen foram descritos como geminicolpados, com seis pares de colpos que estão anastomosados nos polos. Com base na presente análise, os grãos de pólen foram definidos como possuindo pseudopérculos e os colpos não foram caracterizados como geminicolpados nem sincolpados.

As espécies estudadas, que ocorrem na região Sudeste do Brasil, apresentam uma grande variabilidade na morfologia foliar, o que dificulta, sobremaneira, sua identificação com base apenas nessa estrutura vegetativa (Milward-de-Azevedo 2003). A análise dos grãos de pólen empreendida mostrou a possibilidade de utilização de novas características diagnósticas em nível específico, principalmente em relação aos táxons afins e, às vezes, em outros níveis hierárquicos infragenéricos, como seções e séries. No primeiro contexto, pode-se assinalar a grande afinidade entre $P$. misera e $P$. organensis, distintas entre si pelo número de séries da corona e com distribuição geográfica alopátrica no Sudeste brasileiro (Milward-de-Azevedo 2003). No presente estudo, essas espécies também puderam ser distinguidas pelas dimensões dos diâmetros do lúmen dos retículos (tabela 5), além da presença ou não de báculos no interior do lúmen. As demais espécies também apresentaram características diagnósticas em relação à morfopalinologia, embora não sejam taxonomicamente afins.

Com base nos caracteres polínicos, observa-se que as seções Xerogona, Pseudodysosmia e Decaloba podem ser circunscritas pela forma e pelas características da exina (retículos e lúmen). Nesse caso, apesar de compartilharem grãos de pólen com o mesmo tipo de abertura (12-colporados), estas seções diferem da seção Cieca por um conjunto de particularidades mais específicas, referentes à forma do pólen, ao diâmetro do lúmen e à composição dos retículos.

As espécies da seção Decaloba, que compreende o maior número das espécies estudadas, também podem ser reunidas, a priori, com base em caracteres polínicos, nas mesmas séries que compõem atualmente a subdivisão sistemática do gênero como presença ou não de báculos livres no interior dos lumens, comprimento do colpo e diâmetro do lúmen do retículo. Por outro lado, nota-se uma grande fragilidade na circunscrição da seção Cieca, pois as duas espécies analisadas diferenciaram-se, nitidamente, pela presença ou não de cólporos nos grãos de pólen. Essa característica é importante no tratamento taxonômico do grupo. Em obras clássicas e em outras recentemente publicadas, os grãos de pólen da família têm sido normalmente descritos como 6- e 12-colporados (Judd et al. 1999, MacDougal 1994, Presting 1965).

No estudo das espécies de Passiflora subg. Decaloba ocorrentes no Sudeste brasileiro, diversas características polínicas mostraram valor diagnóstico

Tabela 5. Média (em $\mu \mathrm{m}$ ) da medida das camadas de exina e diâmetro do lúmen dos grãos de pólen $(\mathrm{n}=10)$ de espécies de Passiflora subg. Decaloba.

Table 5. Mean $(\mu \mathrm{m})$ of morphometric data of exine and lumen diametre pollen grains $(\mathrm{n}=10)$ Passiflora subg. Decaloba species.

\begin{tabular}{lcccr}
\hline Espécies & Exina & Sexina & Nexina & Lúmen \\
\hline P. capsularis & 2,0 & 1,8 & 0,2 & 9,2 \\
P. misera & 1,8 & 1,7 & 0,1 & 2,7 \\
P. morifolia & 2,0 & 1,9 & 0,1 & 12,0 \\
P. organensis & 2,7 & 1,9 & 0,8 & 6,0 \\
P. pohlii & 1,6 & 1,5 & 0,1 & 2,0 \\
P. suberosa & 2,0 & 1,9 & 0,1 & 2,0 \\
P. tricuspis & 1,5 & 1,4 & 0,1 & 3,7 \\
P. truncata & 1,0 & 0,9 & 0,1 & 1,0 \\
\hline
\end{tabular}


664 M.A. Milward-de-Azevedo et al.: Palinotaxonomia de espécies de Passiflora subg. Decaloba no Sudeste brasileiro

Tabela 6. Medidas $(e m \mu \mathrm{m})$ dos grãos de pólen, em vista equatorial, dos materiais de comparação $(\mathrm{n}=10)$ de espécies de Passiflora subg. Decaloba.

Table 6. Measures $(\mu \mathrm{m})$ of pollen grains, equatorial view, of comparison $(\mathrm{n}=10)$ Passiflora subg. Decaloba species.

\begin{tabular}{|c|c|c|c|c|c|c|}
\hline \multirow[t]{2}{*}{ Espécies } & \multicolumn{2}{|c|}{ Diâmetro polar } & \multicolumn{2}{|c|}{ Diâmetro equatorial } & \multirow[b]{2}{*}{$\mathrm{DP} / \mathrm{DE}$} & \multirow[b]{2}{*}{ Forma } \\
\hline & Faixa de variação & $\overline{\mathrm{x}}$ & Faixa de variação & $\overline{\mathrm{x}}$ & & \\
\hline \multicolumn{7}{|l|}{ P. capsularis } \\
\hline RB 271409 & $62,0-68,0$ & 65,0 & $62,0-69,0$ & 65,0 & 1,02 & prolato-esferoidal \\
\hline IAC 32331 & $48,7-56,2$ & 52,4 & $50,0-53,7$ & 52,2 & 1,00 & esferoidal \\
\hline UEC 98150 & $60,0-65,0$ & 62,2 & $55,0-62,5$ & 57,9 & 1,07 & prolato-esferoidal \\
\hline \multicolumn{7}{|l|}{ P. misera } \\
\hline RB 252079 & $45,0-55,0$ & 50,7 & $43,0-52,0$ & 47,9 & 1,05 & prolato-esferoidal \\
\hline R 50348 & $57,5-62,5$ & 59,0 & $50,0-57,5$ & 53,2 & 1,11 & prolato-esferoidal \\
\hline IAC 2851 & $47,5-60,0$ & 53,0 & $47,5-57,5$ & 50,3 & 1,05 & prolato-esferoidal \\
\hline SP 270253 & $55,0-60,0$ & 58,0 & $50,0-55,0$ & 53,2 & 1,09 & prolato-esferoidal \\
\hline \multicolumn{7}{|l|}{ P. morifolia } \\
\hline UEC 12634 & $70,0-77,5$ & 74,2 & $57,5-72,5$ & 62,7 & 1,18 & subprolata \\
\hline IAC 32621 & $66,1-66,3$ & 66,2 & $50,0-57,5$ & 53,8 & 1,23 & subprolata \\
\hline \multicolumn{7}{|l|}{ P. organensis } \\
\hline RB 354874 & $54,0-61,0$ & 58,9 & $56,0-61,0$ & 58,4 & 1,01 & prolato-esferoidal \\
\hline HB 52166 & $62,5-67,5$ & 64,0 & $52,5-62,5$ & 58,4 & 1,09 & prolato-esferoidal \\
\hline GUA4482 & $62,5-72,5$ & 68,7 & $62,5-70,0$ & 66,2 & 1,04 & prolato-esferoidal \\
\hline \multicolumn{7}{|l|}{ P. pohlii } \\
\hline RB 340719 & $55,0-60,0$ & 57,5 & $49,0-56,0$ & 53,3 & 1,09 & prolato-esferoidal \\
\hline PAMG 44398 & $52,5-58,7$ & 56,4 & $48,7-53,7$ & 51,7 & 1,09 & prolato-esferoidal \\
\hline IAC 18582 & $56,2-62,5$ & 58,9 & $50,0-57,5$ & 54,5 & 1,08 & prolato-esferoidal \\
\hline \multicolumn{7}{|l|}{ P. suberosa } \\
\hline RB 90516 & $75,0-85,0$ & 78,2 & $57,5-75,0$ & 64,0 & 1,22 & subprolata \\
\hline IAC 32542 & $60,0-70,0$ & 63,4 & $50,0-62,5$ & 54,6 & 1,16 & subprolata \\
\hline UEC 22048 & $58,7-67,5$ & 63,9 & $47,5-55,0$ & 50,0 & 1,28 & subprolata \\
\hline \multicolumn{7}{|l|}{ P. tricuspis } \\
\hline ВHCB 64897 & $60,0-67,5$ & 63,4 & $57-5-62,5$ & 60,2 & 1,05 & prolato-esferoidal \\
\hline RB 54025 & $55,0-58,7$ & 57,2 & $48,7-52,5$ & 51,2 & 1,12 & prolato-esferoidal \\
\hline \multicolumn{7}{|l|}{ P. truncata } \\
\hline R 90277 & $45,0-57,5$ & 52,0 & $40,0-52,5$ & 45,9 & 1,13 & prolato-esferoidal \\
\hline GUA 16358 & $47,5-55,0$ & 51,0 & $45,0-55,0$ & 48,7 & 1,05 & prolato-esferoidal \\
\hline
\end{tabular}

específico, como o número de cólporos, os tipos e diâmetros dos lumens dos retículos e o comprimento dos colpos. Outro dado importante que esta análise confirmou para a taxonomia do gênero foi a ocorrência de grãos de pólen colpados, apenas em $P$. suberosa. Entretanto, essa mesma característica não reuniu as espécies da seção Cieca, trazendo dúvidas quanto à classificação proposta por Killip (1938), embora as demais espécies das respectivas categorias infrasubgenéricas não tenham sido estudadas. Deste modo, depreende-se a relevância que a continuação do estudo dos grãos de pólen poderá ter para o entendimento da sistemática da família, inclusive utilizando-se caracteres palinológicos num estudo de análise cladística, a fim de se reavaliar as circunscrições e o arranjo das categorias infragenéricas e, conseqüentemente, um melhor entendimento da monofilia das seções e séries classicamente aceitas.

Agradecimentos - À Capes e ao CNPq pelas bolsas concedidas, respectivamente, à primeira e aos demais autores. À técnica do microscópio eletrônico de varredura, Msc. Noêmia Rodrigues Gonçalves, do Instituto de Biofísica da UFRJ, pela obtenção das eletromicrografias. Aos herbários pelo empréstimo do material para a análise palinológica. 


\section{Referências bibliográficas}

BARTH, O.M. \& MELHEM, T.S. 1988. Glossário ilustrado de palinologia. Editora Universidade Estadual de Campinas, Campinas.

ERDTMAN, G. 1960. The acetolysis method. A revised description. Svensk Botanisk Tidskrift 54:561-564.

GARCÍA, M.T.A., GALATI, B.G. \& ANTON, A.M. 2002. Microsporogenesis, microgametogenesis and pollen morphology of Passiflora spp. (Passifloraceae). Botanical Journal of the Linnean Society 139:383-394.

HOLMGREN, P.K., HOLMGREN, N.H. \& BANETT,L.G. 1990. Index Herbariorum. Part 1: The herbaria of the world. $8^{\text {th }}$ ed., New York Botanical Garden, New York.

HUANG, T.C. 1968. Pollen grains of Formosan plants. Taiwania 14:133-270.

HUYNH, K.L. 1972. Étude de l'arrangement du pollen dans la tétrade chez les angiosperms sur la base de dones cytologiques. IV. Le genre Passiflora. Pollen et Spores 14:51-60.

JUDD, W.S., CAMPBELL, C.S., KELLOG, E.A. \& STEVENS, P.F. 1999. Plant Systematics: a phylogenetic approach. Sianuer Associates, Massachusetts.

KILLIP, E.P. 1938. The American Species of Passifloraceae. Publication Field Museum of Natural History - Botanical Series 19:1-613.
MACDOUGAL, J.M. 1994. Revision of Passiflora subgenus Decaloba section Pseudodysosmia (Passifloraceae). Systematic Botany Monographs 41:1-146.

MILWARD-DE-AZEVEDO, M.A. 2003. Passiflora subgênero Decaloba (DC.) Rchb. (Passifloraceae) no Sudeste do Brasil. Dissertação de mestrado, Museu Nacional - Universidade Federal do Rio de Janeiro, Rio de Janeiro.

PRESTING, D. 1965. Zur morphologie der pollenkörner der Passifloraceen. Pollen et Spores 7:193-247.

PUNT, W., BLACKMORE, S., NILSSON, S. \& LE THOMAS, A. 1999. Glossary of pollen and spore terminology. http:/ /www.biol.ruu.nl/ palaeo/glossary/glos-int.htm. (acesso em 18/04/2003).

ROUBIK, D.W. \& MORENO, J.E.P. 1991. Pollen and Spores of Barro Colorado. Missouri Botanical Garden, Saint Louis.

SALGADO-LABOURIAU, M.L. 1973. Contribuição à palinologia dos cerrados. Academia Brasileira de Ciências, Rio de Janeiro.

SPIRLET, M.L. 1965. Utilisation taxonomique des grains de pollen de Passifloracées. Pollen et Spores 7:249-301.

VIEIRA, S. 1981. Introdução à Bioestatística. Editora Campus, Rio de Janeiro. 